\title{
Flutter analyses of complete aircraft based on hybrid grids and parallel computing
}

\author{
ZHENG GuanNan ${ }^{1}$, YANG GuoWei ${ }^{1 *} \&$ QIAN Wei ${ }^{2}$ \\ ${ }^{1}$ Key Laboratory for Mechanics in Fluid Solid Coupling Systems Institute of Mechanics, Chinese Academy of Sciences, \\ Beijing 100190, China; \\ ${ }^{2}$ Shenyang Aircraft Design and Research Institute, Shenyang 110000, China
}

Received May 9, 2012; accepted October 9, 2012; published online December 5, 2012

\begin{abstract}
A tightly coupled method was developed to analyse aeroelasticity by constructing subiterative schemes for fluid and structural equations of motion, respectively. With MPI partition parallel computing, the fluid was solved by Navier-Stokes equations based on hybrid grids. A new unstructured background grid deformtion method was used for the CFD grid deformation. The transonic flutter wind tunnel model of a complete aircraft was simulated to validate the developed method. The flutter characteristics of the aircraft was analysed and compared with the test results. It indicates that the devoloped method has a relatively higher precision and can be used for aeronautical engineering application.
\end{abstract}

CFD, CSD, aeroelasticity, coupled method, flutter, MPI

Citation: Zheng G N, Yang G W, Qian W. Flutter analyses of complete aircraft based on hybrid grids and parallel computing. Sci China Tech Sci, 2013, 56: 398-404, doi: 10.1007/s11431-012-5073-5

With the rapid growth of domestic economy, a great opportunity has been appearing for the entire aviation industry in both civil and military aircraft. The flutter property is one of the key problems in aircraft design process, and has attracted more and more attentions. Before the 1990s, the flutter boundaries were mainly predicted by solving linearized unsteady aerodynamic equations coupled with linearized generalized structural dynamic equations. However, as flight Mach number is within the transonic range, the emergence of shockwaves and flow separation flows due to the interaction of shock and boundary layer can lead to highly nonlinear flow field, thus the fluid linearized assumption is no longer valid. Therefore, Euler or Navier-Stokes equations need to be used to obtain the the nonlinearied unsteady aerodynamic forces [1].

On the basis of our previous investigations, a parallel

*Corresponding author (email: gwyang@imech.ac.cn) computing method for Navier-Stokes equations on flutter analyses was developed based on hybrid grids in this paper. In this method, both fluid and structural equations are constructed with the dual-time iterative schemes [2]. Based on our investigation [3], the time-marching DP-LUR scheme for fluid was constructed into sub-iteration scheme and the structural equation of motion was discretized by the method of ref. [4]. A tightly coupled aeroelastic calculation method based on hybrid grids and parallel computing was developed. In the flutter calculation process, due to the structural deformation with time, adaptive grids need to be developed at each time step. In this paper, the real-time deformation of the hybrid grids is obtained by the spring network method of background grid method put forward by us [5]. In the previous flutter investigation, the structural deformation only in the primary direction was taken into consideration and the deformation of other two directions was omitted. In this paper, the structural deformations of all three directions 
are considered, so that the structural deformation is much closer to the actual one. At present, the flutter calculation coupled CFD with CSD still faces the huge challenge of time consumption, so the CFD calculation that takes up most of the computational time is paralleled by MPI [6] which reduces the computational time greatly and improves the efficiency of solving engineering problems. The accuracy and applicability of the developed method has been verified by flutter analyses of the standard wing model 445.6 [7] and a vertical tail with rudder control surface [8].

In the paper, we firstly validated and verified the developed method through analyzing the subsonic flutter characteristics of a complete aircraft wind tunnel model and comparing the results with those of NASTRAN software. Then the transonic flutter characteristics of the aircraft model were simulated. Finally, by the comparison of calculation results with the wind tunnel experimental results, the transonic flutter predicted precision for complex aircraft configuration was further verified. It provided a reference for further engineering applications.

\section{Solution of generalized structural equations}

Based on the Rayleigh-Ritz method, the generalized structural equations of motion could be expressed as the following second-order ordinary differential equations:

$$
\begin{gathered}
\boldsymbol{M} \ddot{\boldsymbol{q}}(t)+\boldsymbol{D} \boldsymbol{q}(t)+\boldsymbol{K} \boldsymbol{q}(t)=\boldsymbol{F}(t), \\
\boldsymbol{w}(x, y, z, t)=\sum_{i=1}^{N} q_{i}(t) \phi(x, y, z), \\
\boldsymbol{F}(t)=\sum_{i=1}^{3} \oiint \Delta f_{i}(x, y, z, t) \phi_{i}(x, y, z) \mathrm{d} s,
\end{gathered}
$$

where $\boldsymbol{w}(x, y, z, t)$ represents the structural deformation of the aircraft surface, and $\boldsymbol{q}(t)$ the generalized displacement, both of them describe the time history of the aircraft structural deformation. $\boldsymbol{F}(t)$ represents the generalized aerodynamic force. $\boldsymbol{M}, \boldsymbol{D}$ and $\boldsymbol{K}$ are the generalized mass, the structural damping and the stiffness matrix, respectively. These physical quantities can be calculated by finite element analyses and NASTRAN software, or can be obtained by experimental measurement, which are independent of flow field but dependent on the characteristics of aircraft structural and mass distribution.

To solve the generalized structural equations of motion, we can assume $\boldsymbol{S}=\{\boldsymbol{q}(t), \dot{\boldsymbol{q}}(t)\}$; then the eq. (1a) may be written as the linear equation group:

$$
\frac{\mathrm{d} \boldsymbol{S}}{\mathrm{d} t}=\boldsymbol{P}=\left[\begin{array}{c}
0 \\
\boldsymbol{F}(t) / M
\end{array}\right]-\left[\begin{array}{cc}
0 & -1 \\
\boldsymbol{K} / \boldsymbol{M} & \boldsymbol{D} / \boldsymbol{M}
\end{array}\right] \boldsymbol{S} .
$$

For the CFD/CSD coupling computation, the fully coupled method, the tightly coupled method and loosely coupled method were developed $[9,10]$.

Generally, eq. (2) could be solved by using classical fourth-order Runge-Kutta method. However, the values of $n+1$ time step is only associated with current time step of $n$; even if the fluid solver is with a sub-iteration, the solution of generalized structural equations of motion can be obtained only after the finish of flow field sub-iteration. So this method belongs to a loosely coupled method. Using the above loosely coupled method, regardless of the flow field and structure calculations with high time accuracy schemes, the time accuracy of the coupled calculation is still only the first order. Therefore, eq. (2) would be written as the following sub-iteration form:

$$
\begin{gathered}
{\left[\begin{array}{cc}
1 & -\phi^{i} \Delta t \\
\phi^{i} \Delta t \boldsymbol{K} / \boldsymbol{M} & 1+\phi^{i} \Delta t \boldsymbol{D} / \boldsymbol{M}
\end{array}\right] \Delta \boldsymbol{S}} \\
=-\phi^{i}\left\{(1+\phi) \boldsymbol{S}-(1+2 \phi) \boldsymbol{S}^{n}+\phi \boldsymbol{S}^{(n-1)}+\Delta t \boldsymbol{P}^{p}\right\}, \\
\Delta \boldsymbol{S}=\boldsymbol{S}^{(p+1)}-\boldsymbol{S}^{(p)}, \phi^{i}=\frac{1}{(1+\phi)} .
\end{gathered}
$$

When $\phi=0.5, \quad p \rightarrow \infty$, the time accuracy of eq. (3) is second-order. If the sub-iteration is also used for the fluid solution, in each sub-iteration step, the flow and the structural deformation are solved contemporarily, which is a tightly coupled method. When the number of sub-iteration step tends to be infinite, the time accuracy of coupled calculation is second order.

\section{Solution of fluid equations}

In the Cartesian coordinates, the three-dimensional NavierStokes equations can be written as

$$
\partial_{t} Q+\partial_{x} E+\partial_{y} F+\partial_{z} G-\partial_{x} E_{v}-\partial_{y} F_{v}-\partial_{z} G_{v}=S_{G C L},
$$

where $S_{G C L}$ is the geometric conservation item considering the effect of grid deformation. In this paper, the DP-LUR implicit time-marching scheme with sub-iteration was used, which can be written as

$$
D^{-1} \Delta Q=-\phi^{i}\left\{\begin{array}{l}
(1+\phi) Q^{p}-(1+2 \phi) Q^{n}+\phi Q^{n-1} \\
-\Delta t\left(\delta_{x} E^{p}+\delta_{y} F^{p}+\delta_{z} G^{p}\right) \\
+\Delta t\left(\delta_{x} E_{v}^{p}+\delta_{y} F_{v}^{p}+\delta_{z} G_{v}^{p}\right)
\end{array}\right\} .
$$

The difference compared with the standard DP-LUR scheme is that the variable of $\phi^{i}$ is added, which could be written as

$$
\begin{aligned}
& D=\bar{\rho} I+\phi^{i} \Delta t \sum_{j(i)} A_{i j}, \\
& \bar{\rho}=1+\phi^{i} \Delta t \sum_{j(i)} \bar{\rho}_{i j}, \\
& \phi^{i}=1 /(1+\phi), \Delta Q=Q^{p+1}-Q^{p} .
\end{aligned}
$$

In the sub-iterative process, eqs. (3) and (5) are solved 
contemporarily, a so-called tightly coupled aeroelastic analysis method is obtained. Here, the convective items of eq. (5) were discretized with the improved HLLEW scheme [11] and the viscous terms were discretized with the second-order central scheme. The improved HLLEW scheme can automatically revert to the Roe scheme in isentropic flow region and can revert to the standard HLLEW scheme in the flow region of the large entropy fluctuations. In this way, the non-physical oscillations in the shock region with Roe scheme and the too large artificial viscosity in the continuous flow region with HLLEW scheme can be avoided. Thereby the calculation accuracy of overall flow field will be improved. The $k-\omega$ two-equation turbulence model was used to simulate the turbulence flow, which has the higher turbulence solution accuracy and has been applied extensively. More important is that the model needs no distance function, which could save much time for the flutter calculation having dynamic grid deformation and can improve the computational efficiency.

\section{Grid deformation and parallel computing}

Because the hybrid grids with the tetrahedral, prism, hexahedral and pyramid cells are used, the traditional algebraic deformation method for the structured grid cannot be used and the spring network method [5] for unstructured grid cannot be used for the deformation of hybrid grids either. In this paper, a hybrid grid deformation method by using unstructured background grid deformation was developed. In this method, a sparse unstructured background grid is contemporarily generated, which is deformed with the spring network method. Then the deformation displacement of the hybrid CFD grid is obtained by the algebraic interpolation based on the deformation displacement of the background grid. Its detail can be found in ref. [12].

The parallel computing method mainly contains parallel partition and parallel realization. In this paper, the parallel computing was realized based on MPI message transfer method, so the grid partition and parallel realization have the same importance.

The MPI message transfer method is different from the memory-sharing parallel method which is suitable for the parallel computing of large-scale distributed cluster computers. However, it is strongly dependent on the communication technique and communication efficiency. Therefore, under the same calculation environment, the parallel efficiency can be well improved if the quantity of communication, which is strongly dependent on parallel partition, can be made as less as possible. At present, the $p$-metis multilevel $K$-way hyper-graph partition method [13] of University of Minnesota is used, which is a relatively ideal partition method.

The parallel algorithm is realized by MPI message transfer. Improving communication efficiency and reducing communication jam phenomenon are the most effective means to improve the parallel efficiency for large-scaled parallel calculation. In the paper, the staining layered communication method developed by us [6] is used, which has the advantages such as higher communication efficiency, lower communication amount, and no communication jam. The detail can be found in ref. [6].

\section{Numerical examples and analysis}

For the simple configuration, the developed method has been verified by the aeroelastic standard model wing 445.6 [8], which behaves a higher accuracy for the flutter simulation of subsonic and transonic flows. In the paper, the numerical accuracy of the flutter solver was further verified by simulating the flutter characteristics for the wind tunnel model of the complete aircraft.

The main aim of this paper is to study the flutter characteristics of the complete aircraft model. To improve the accuracy of calculation, the hybrid grids with boundary layer grid for the solution of Navier-Stokes equations is used. To select the hybrid grid size, after a lot of comparative investigations we found that the ten million hybrid grid could basically meet the accuracy requirements of the calculation. The surface grids of the aircraft are shown in Figure 1(a) and (b). The number of the triangle cells on the aircraft surface is 555190, and 9 layered grids are for the boundary layer simulation. The thickness of the first boundary layer grid could satisfy the $Y+$ requirement, and all the values of

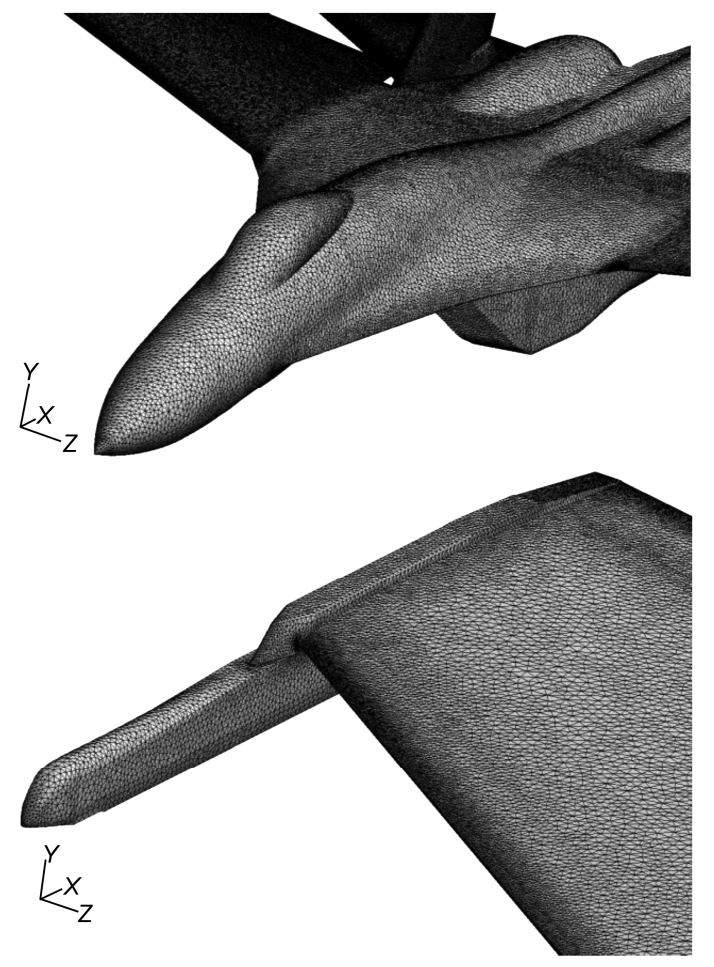

Figure 1 Surface grids on a aircraft model. (a) Surface grid on the head of a aircraft model; (b) surface grid on the pylon of wing tip. 
$Y+$ were between 0.5 to 1.0 . The number of tetrahedron gird cells is 10094920 and the total number of the hybrid grids is 15091630 , which reaches the accuracy requirement of flutter calculation.

The structural modal data of the model were provided by the Aircraft Design Institute. The overweight model was considered to make the divergent flutter of the aircraft model easier. The structural overweight model includes the first sixth-order modes, whose oscillating shapes are shown in Figure 2. The traditional flutter calculation considers only primary directional structural deformation. In the paper, three-dimensional structural deformations of the aircraft are considered which include body, wing, horizontal tail, vertical tail, and engine intake. Because the key objective of this paper is to study the flutter characteristics of the complete aircraft, the geometries of the rudders of vertical and horizontal tails were relevantly simplified.

Generally, the generalized displacement time curve of the structural deformation is calculated by the coupling simulation of CFD and CSD, however, the aircraft design units need to know the flutter critical boundaries, so a series of generalized displacement time curves are calculated. In common flight, the safety of structural dynamic strength of the aircraft is guaranteed and structural vibration basically tends to converge, then we need to change some parameters to make the vibration to diverge. Currently, the variable structural stiffness method, the variable flow density method and the variable flow velocity method are commonly used for the determination of flutter boundary. In the above methods, the Reynolds numbers are same, and equal to the experimental Reynolds number. In fact, it is easy to deduce

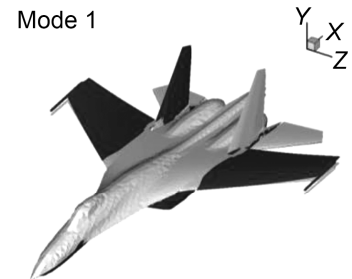

(a)

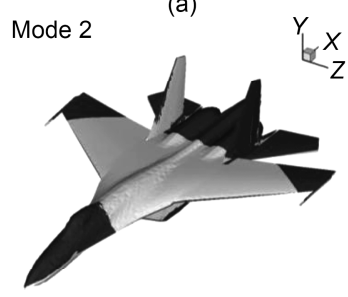

(b)

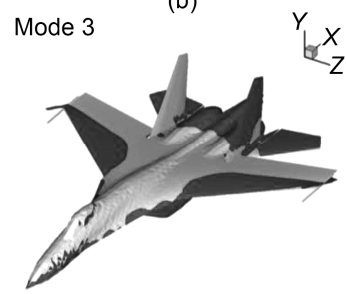

(c)

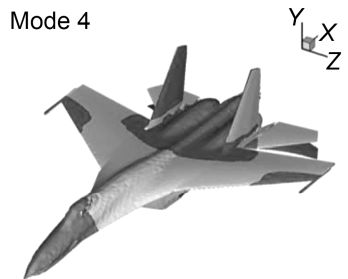

(d)

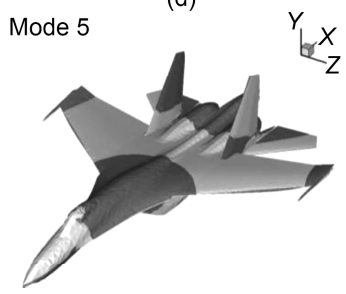

(e)

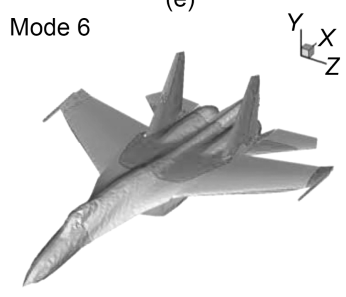

(f)
Figure 2 The Sixth-order mode shape of the aircraft model. that both the variable structural stiffness method and the variable flow velocity method are equivalent. In the paper, only the last two methods are used.

Because the number of hybrid grids is larger and the unsteady flow computation of the flutter is very expensive too, only two typical calculation conditions of Mach numbers 0.2 and 0.9 were considered in this paper. The flutter speed of the model was verified by the calculation of Mach 0.2 using the variable flow velocity method. The comparisons of the experiments and numerical simulations were given for Mach number of 0.9 with the variable flow density method and the variable flow velocity method, respectively. Figures 3-5, show the generalized displacement time curves for Mach number 0.2 by the variable flow velocity method and Mach number 0.9 by both methods, respectively.

By the variable flow velocity method, the simulation of Mach number 0.2 was to validate the developed method with the NASTRAN. The variables in Figure 3 were nondimensionlized with the incompressible results of the NASTRAN software. It is indicated that the accuracy of the developed method could meet the requirement of flutter calculation for engineering application. The flutter speed with the developed method is about 1.097 times the result of NASTRAN.

Because the structural mass distribution of the aircraft model was overweight, the phenomenon of flutter divergence was recorded easier in the wind tunnel tests. At the transonic flow of Mach number 0.9, in order to verify the accuracy of the developed method, the variable flow density method was used to completely simulate the real wind tunnel testing process. For comparison of the experimental and the simulated results, the flow density in Figure 4 was nondimensionlized with the flutter density of wind tunnel tests. In the simulations, the Mach number and the free-stream velocity were fixed, and the values of flow density were taken as six different values between 0.985 and 1.230. The simulation results are shown in Figure 4. Because of the limitations of the paper, Figure 4 only shows the four generalized displacement time histories with different flow densities. Compared with the experimental results, the difference of the flutter speed is about $9.5 \%$. Because the other parameters were taken as the same values of wind tunnel tests, the difference of the flutter dynamic pressure is about $9.5 \%$. To consider systematic errors in this case, the developed method could meet the requirement of engineering applications.

To further study the accuracy of the developed method, the variable flow velocity method was also used to simulate the flutter characteristics of the aircraft. In this case, the Mach number and the flow density were fixed, and the values of free-stream velocity were taken as four different values. The simulation results were shown in Figure 5. The difference of flutter speed compared with the experimental results was about $5.4 \%$, which is about 1.027 times the test flutter speed. Compared with the variable flow density 


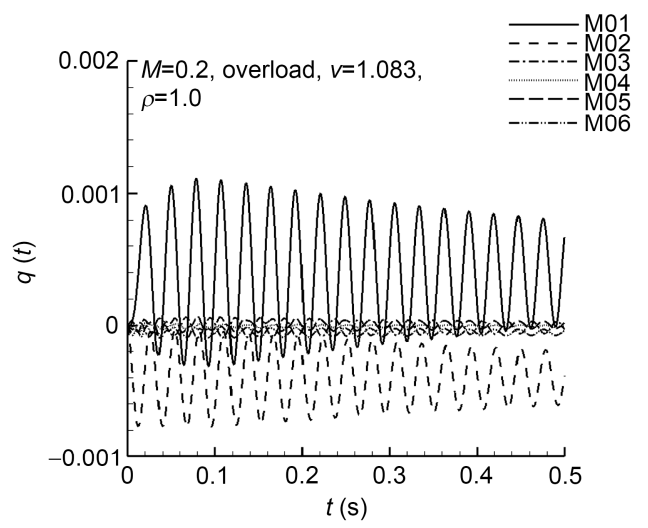

(a)

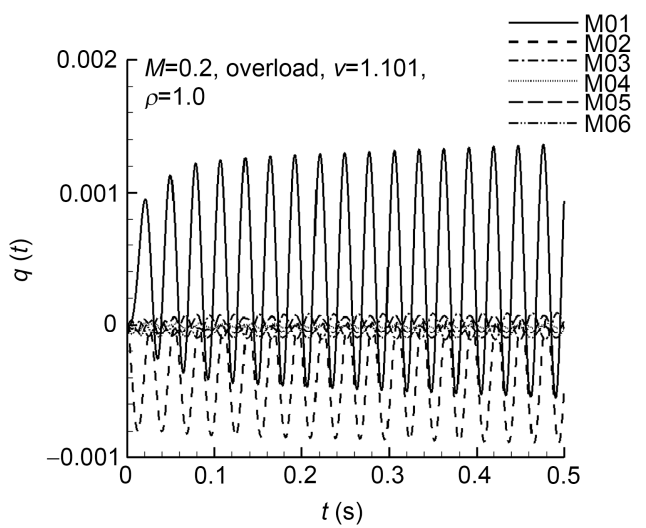

(c)

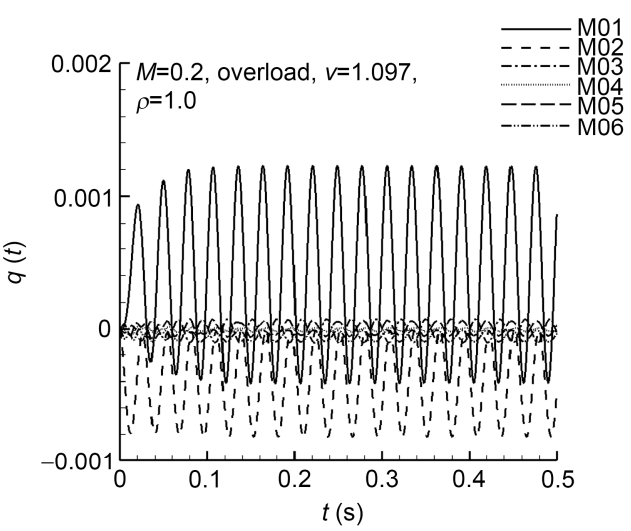

(b)

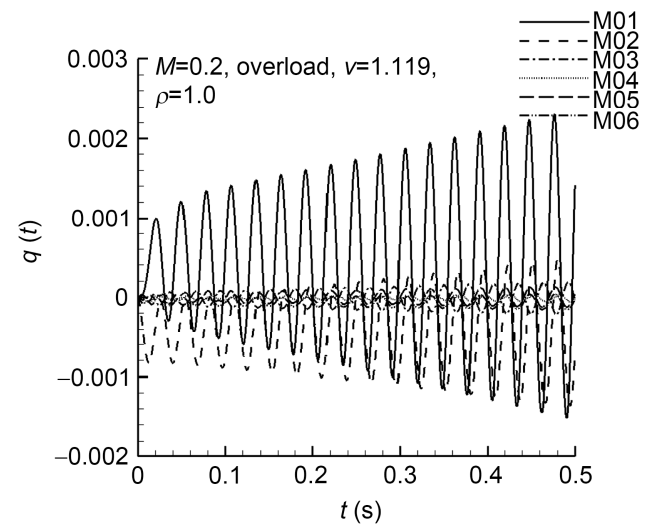

(d)

Figure 3 Generalized displacement time histories for different free-stream velocities at Mach=0.2.

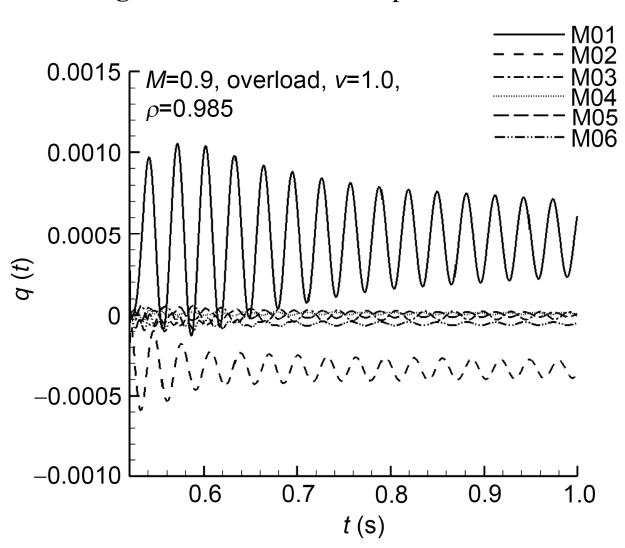

(a)

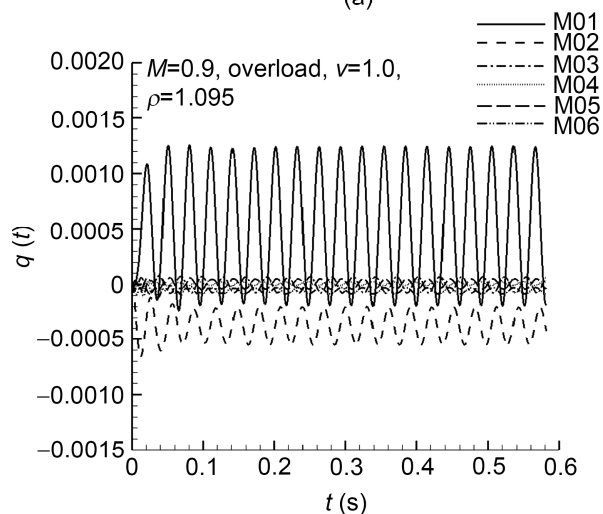

(c)

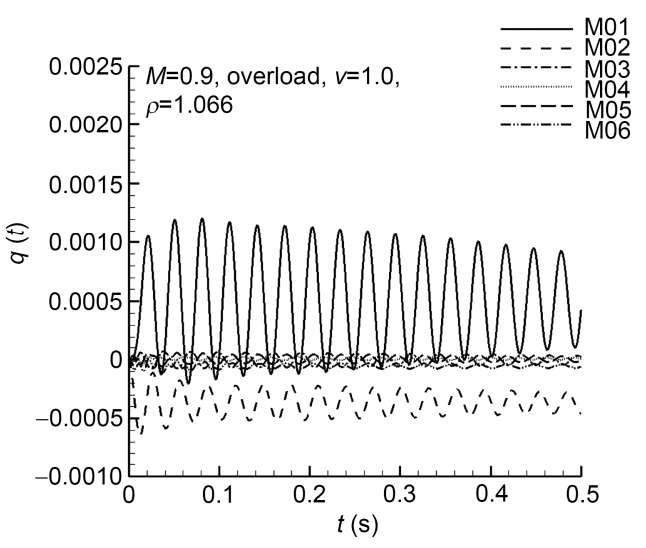

(b)

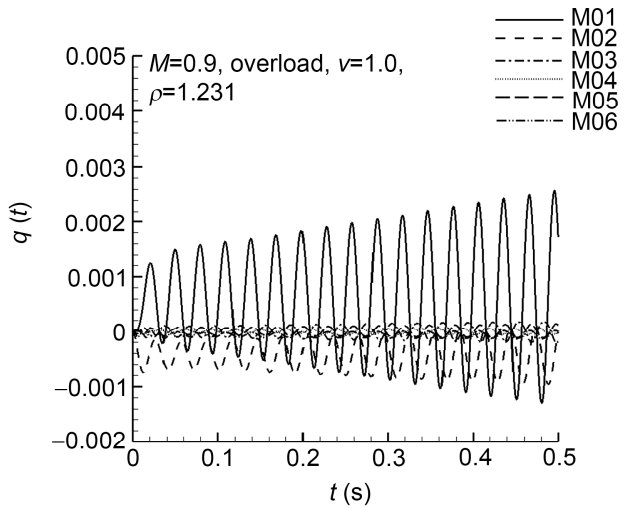

(d)

Figure 4 Generalized displacement time histories with different free-stream densities at Mach=0.9. 


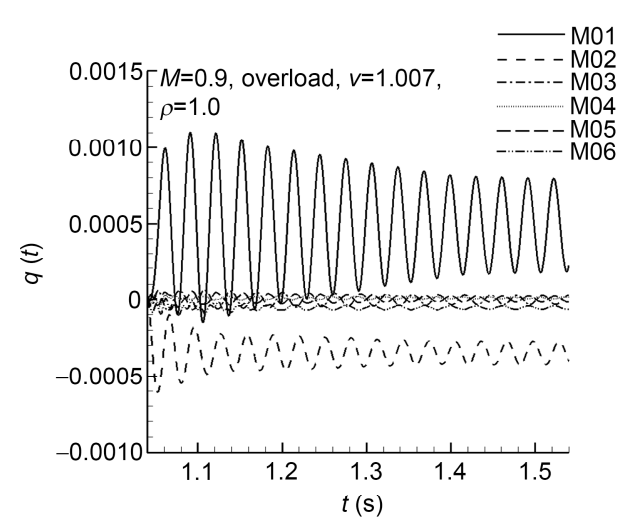

(a)

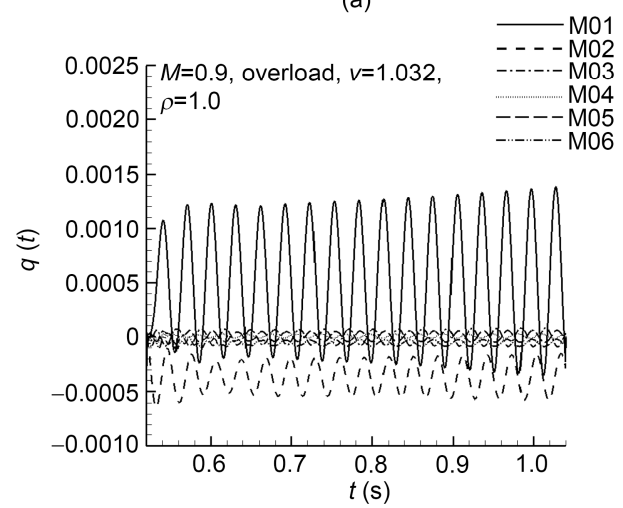

(c)

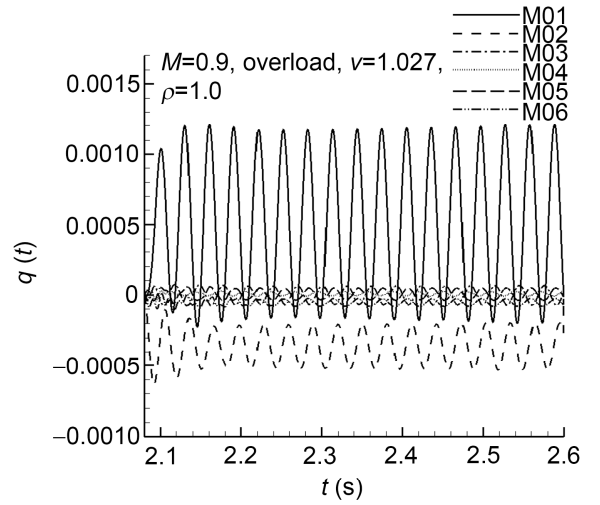

(b)

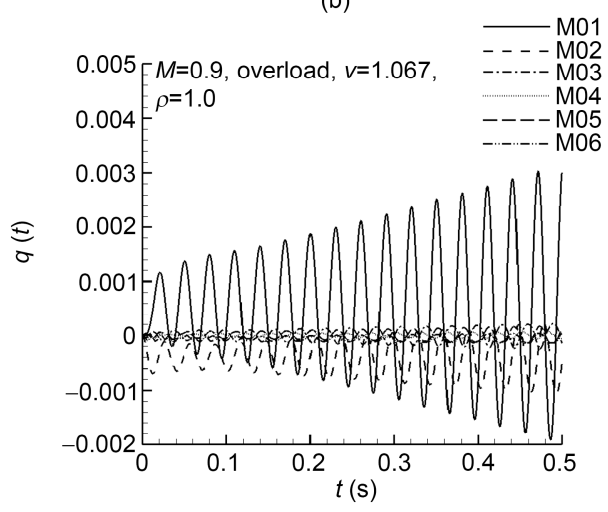

(d)

Figure 5 Generalized displacement time histories with different free-stream velocities at Mach=0.9.

method, the flutter speed is much closer to that of the experiment. Because other variables of flutter calculation were taken as the same values of the variable flow density method, the variable flow velocity method is shown to be much suitable for the flutter analysis.

To study the accuracy of the different coupled method, the flutter characteristics of the aircraft were calculated by using the tightly coupled method $(\phi=0.5)$, the tightly coupled method $(\phi=0.0)$ and loosely coupled method. In this case, the Mach number, the Reynolds number, the flow density and the flow velocity all were fixed. The simulation results were shown in Figure 6. Because all variables of flutter calculation were taken as the same values of the experimental result, the tightly coupled method $(\phi=0.5)$ is shown to be the higher accuracy. At the same time, the tightly coupled method $(\phi=0.0)$ and loosely coupled method are the same accuracy. The other simulation results were shown in Figure 7. In this case, except the flow velocity and density, the Mach number, Reynolds number and pressure of the free-stream are the same, and the free-stream pressure

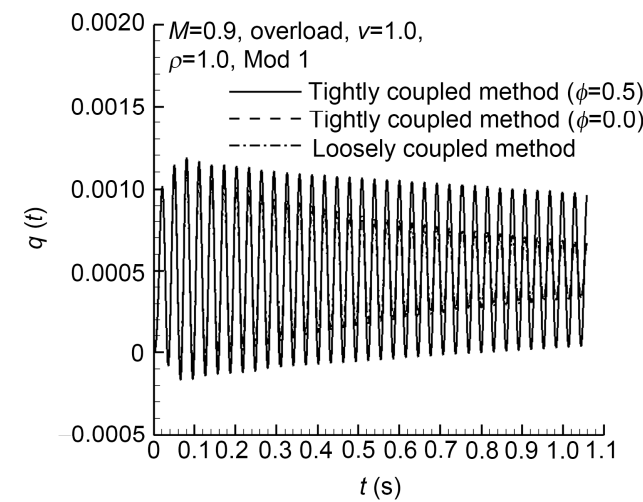

(a)

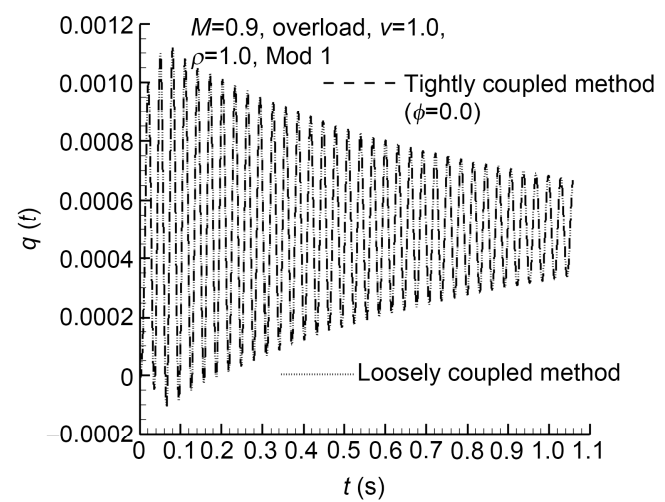

(b)

Figure 6 Generalized displacement time histories with different coupled methods at Mach=0.9. 


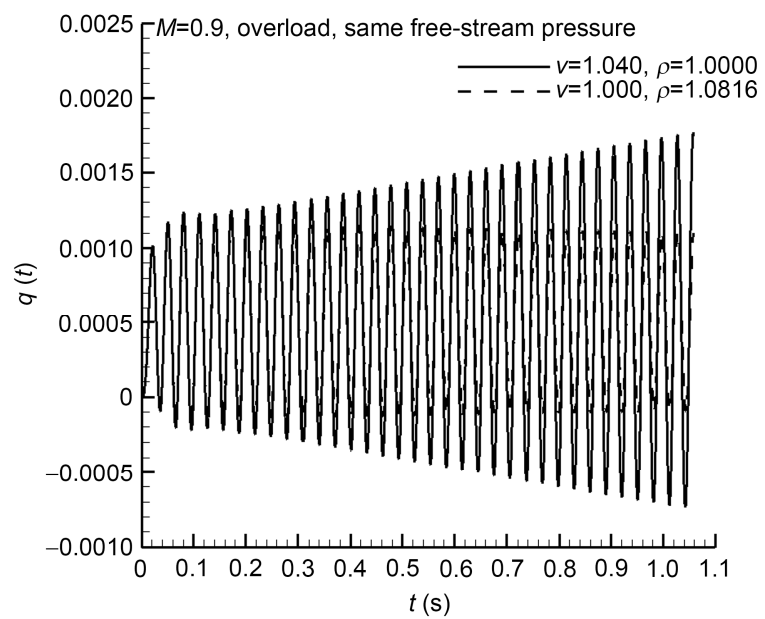

Figure 7 Generalized displacement time histories with same free-stream pressure at $\mathrm{Mach}=0.9$.

is bigger than experimental result. Based on the result, the variable flow velocity method is also shown to be the higher accuracy.

\section{Conclusion}

From the above calculations, for the lower Mach number flow of 0.2 , the flutter results agree very well with those of NASTRAN software. For the transonic Mach number of 0.9 , the difference between calculation by the variable density method and experimental results is about $9.5 \%$, and it is about $5.4 \%$ by the variable velocity method. The numerical results have indicated that the developed method can ana- lyze the flutter characteristics of complex geometric configurations and have a higher numerical accuracy. The method can be applied to engineering applications.

1 Liang Q, Yang Y N, Ye Z Y. Analysis of airfoil flutter characteristic (in Chinese). J Northwest Polytech Univ, 2001, 19: 341-344

2 Alonso J J, Jameson A. Fully-implicit time-marching aeroelastic solutions. AIAA Paper 94-0056, 1994

3 Yang G W, Shigeru S, Nakamichi J. Aileron buzz simulation using an implicit multi-block aeroelastic solver. J Aircraft, 2003, 40: 580589

4 Gordiner R E, Melcille R B. Transonic flutter simulation using an implicit aeroelastic solver. J Aircraft, 2000, 37: 872-879

5 Zheng G N, Yang G W. Three-dimensional dynamic unstructured grid method (in Chinese). In: Thirteenth National Conference of Shock and Shock Tube, 2008. 516-526

6 Zheng G N, Deng S C, Han T L, el al. An implicit parallel computing method based on the Navier-Stokes equations with hybrid grids (in Chinese). Chin J Appl Mech, 2011, 28: 211-218

7 Yates E C. AGARD standard aeroelastic configurations for dynamic response I-wing 445.6. AGARD Report No.765, 1988

8 Zheng G N, Yang G W, Qian W. Flutter analyses based on hybrid grids and parallel computing (in Chinese). In: Eleventh National Conference of Aeroelasticity, 2009. 286-293

9 An X M, Xu M, Chen S L. Analysis for second order time accurate CFD/CSD coupled algorithms (in Chinese). Acta Aerodynam Sinica, 2009, 5: 547-552

10 Farhat C, Zee K G D, Geuzaine P. Provably second-order timeaccurate loosely-coupled solution algorithms for transient nonlinear computational aeroelasticity. Comp Meth Appl Mech Eng, 2006, 195: 1973-2001

11 Obayashi S, Guruswamy G P. Convergence acceleration of a navierstokes solver for efficient static areoelastic computations. AIAA J, 1995, 33: 1134-1141

12 Zheng G N, Yang G W. Hybrid grid deformation method based on background grid (in Chinese). J Vib Eng, 2011, 24: 473-481

13 Karypis G, Kumar V. Multilevel $k$-way hypergraph partitioning. Tech Report TR 98-036. University of Minnesota: Department of Computer Science, 1998 